\title{
Development, testing and demonstration of a portable submersible miniature particle imaging velocimetry device
}

\author{
H M Tritico ${ }^{1,2}$, A J Cotel ${ }^{1}$ and J N Clarke ${ }^{1}$ \\ ${ }^{1}$ Department of Civil and Environmental Engineering, University of Michigan, Ann Arbor, \\ MI 48109, USA \\ ${ }^{2}$ School of Natural Resources and the Environment, University of Michigan, Ann Arbor, \\ MI 48109, USA \\ E-mail: acotel@umich.edu
}

Received 30 January 2007, in final form 19 April 2007

Published 11 July 2007

Online at stacks.iop.org/MST/18/2555

\begin{abstract}
A portable underwater particle image velocimetry (PIV) device has been developed, tested and demonstrated. The underwater PIV uses a $532 \mathrm{~nm}$ battery-powered $90 \mathrm{~mW}$ continuous laser. The laser beam is pulsed via a camera-synchronized chopper wheel. Images were recorded using a 1 megapixel black and white 10-bit CCD battery-powered camera controlled via a PCMCIA frame grabber card connected to a laptop computer. The system was validated against a standard laboratory PIV for average velocities up to $15 \mathrm{~cm} \mathrm{~s}^{-1}$ downstream from a $1.6 \mathrm{~cm}$ circular cylinder. The average vorticities calculated between the two systems were similar with a maximum difference of $3.6 \%$. The average velocities were also similar with the largest difference occurring at the slowest flow recorded (difference of $0.5 \mathrm{~cm} \mathrm{~s}^{-1}$ ), resulting in a $9.4 \%$ difference. The maximum eddy size was comparable between the two systems with an average error of $4 \%$. The system was field tested in the Huron River, Michigan downstream from a $1.2 \mathrm{~cm}$ diameter submerged limb. Mean velocities and standard deviations were comparable to acoustic Doppler velocimeter data. This paper presents the first published subsurface PIV data from a fluvial environment, demonstrating potential applications for a number of ecological and geomorphological studies.
\end{abstract}

Keywords: particle image velocimetry, PIV, underwater, river, lake, field, miniature

(Some figures in this article are in colour only in the electronic version)

\section{Introduction}

Eddy dynamics are expected to affect ecosystem function through mixing and momentum exchange processes ranging from predator-prey interactions to sediment transport capacities. There have been an increasing number of calls for studies which investigate the spatial scale of turbulence in natural aquatic environments (Landry et al 1995, Nikora et al 2003, Standen et al 2004). The most common device currently used for the investigation of turbulence in the natural aquatic environment is the acoustic Doppler velocimeter (ADV). ADVs provide high-resolution temporal velocity data at a single point in the flow stream. They therefore provide excellent temporal resolution but spatial investigations must either work within the constraints of Taylor's frozen turbulence approximation or utilize arrays of ADVs which collect velocity data simultaneously (Roy et al 2004).

Successful attempts have been made in the past at developing submersible particle image velocimetry (PIV) systems but these systems have not been developed in such 

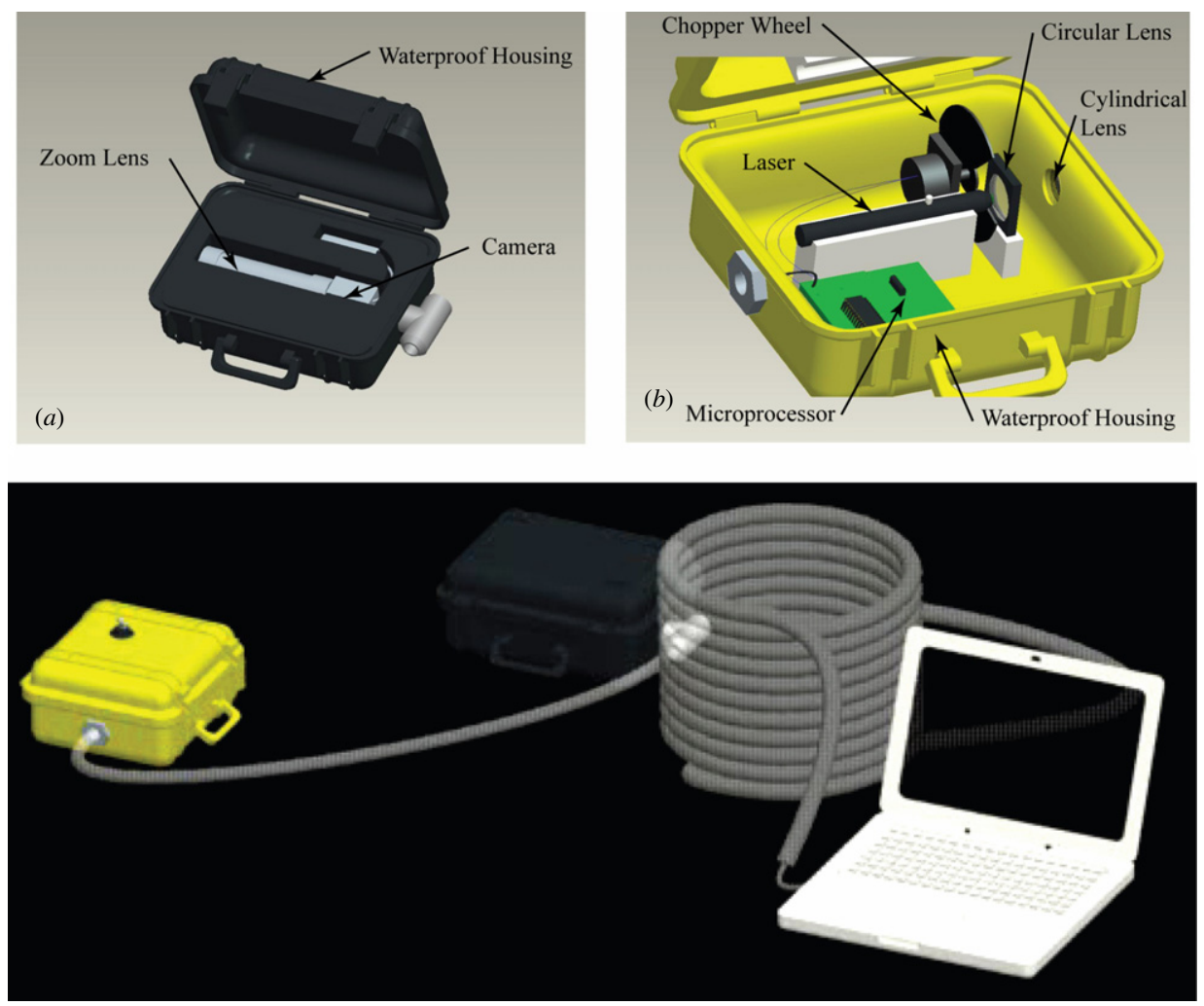

(c)

Figure 1. System schematic: $(a)$ camera system and housing; $(b)$ laser system and housing; and $(c)$ system overview including laptop and connecting cables.

a way that they can be easily transported or set up in natural environments. Submersible PIV systems have been developed to examine the bottom boundary layer of the ocean by Bertuccioli et al (1999) and Nimmo-Smith et al (2002). The PIV system used in these experiments was powered by ship's main power and lowered to the sea floor using on-board cranes. The system utilized a $1024 \times 1024$ CCD camera (Kodak Megaplus XHF) at $30 \mathrm{fps}$ in conjunction with a $350 \mathrm{~mJ}$ pulsed laser (Cynosure Inc.) operating at a wavelength of $594 \mathrm{~nm}$. The laser light was supplied, via fibre optics, to the bottom of the ocean along with the camera system in a waterproof enclosure. The illuminated measurement area was approximately $20 \mathrm{~cm} \times 20 \mathrm{~cm}$ (Bertuccioli et al 1999). Several years later, they re-designed the system to record an illuminated area of $35 \mathrm{~cm} \times 35 \mathrm{~cm}$. This was done by using two $2048 \times 2048$ CCD cameras operating simultaneously (Nimmo-Smith et al 2002). Onboard the ship was a desktop computer with an Alacron frame grabber to store the gathered data. The largest reported speed was approximately $17 \mathrm{~cm} \mathrm{~s}^{-1}$. Alternatively, miniature PIV systems have also been developed by Chetelat et al (2001) and others for the investigation of microscopic flow; however these systems were not designed for field use. We have successfully developed a PIV system which is both submersible and portable for a specific field use, while keeping the cost and complexity of the system minimum.

\section{System overview}

The newly developed PIV system works as follows. A 1 megapixel high-resolution CCD camera collects images and outputs a TTL signal which is fed into a programmable microprocessor board (figure $1(a)$ ). This signal lets the microprocessor know when the images are being captured allowing for synchronization between the camera and a laser chopper wheel (figure 1(b)). The chopper wheel chops the continuous emission from a hand-held laser system into short asynchronous pulses which illuminate the flow in short durations at short pulse separation times. The pulse width and separation can be varied by changing the aperture and rotational velocity of the wheel, for the validation presented below pulse durations, and pulse separations ranged from 6 to $20 \mathrm{~ms}$. The short pulse durations avoid particle streaking in the images while the short pulse separation times reduce the distance particles travel between images. Reducing the distance particles travel between images allows the crosscorrelation integration windows to be smaller and reduces the frequency of particles moving into or out of the laser sheet (ghost particles) (Raffel et al 1998). The laser and timing systems are powered by on-board $3 \mathrm{~V}$ and $9 \mathrm{~V}$ batteries which were changed daily to ensure maximum and consistent power supply. Both systems have on/off switches to allow for reduced power demand. The laser and camera system housings have a combined volume of $0.017 \mathrm{~m}^{3}$ and weigh $7.5 \mathrm{~kg}$, which allow for easy transport and set-up.

\subsection{Camera system}

A UniqVision UP-1830 10-bit black-and-white CCD camera was modified in two ways for PIV data collection and used in this system. The first camera modification enabled image data 
which have been transferred to the laptop to be deleted rapidly (within $1 \mu \mathrm{s}$ ) in order to quickly free space for collection of the second laser pulse image (General Pixels 2000, UniqVision email communication). This modification allows for shorter pulse separation times leading to an increased range of flows which can be captured using PIV analysis (Gharib and Dabiri 2000, Dabiri 2003). The second modification changed the TTL output to be a continuous $+5 \mathrm{~V}$ signal interrupted by a $2 \mathrm{~ms}, 0 \mathrm{~V}$ signal $31 \mu$ s after the optical camera shutter opens, allowing for synchronization of the image capture with laser pulsing. This specification allowed for synchronization between the camera and the laser chopper wheel. The camera captures 1 megapixel images at a rate of $30 \mathrm{fps}$. The images are transmitted via a $10 \mathrm{~m}$ camera-link cable to a PCMCIA frame grabber card. The user, either in a boat or on shore, controls the image acquisition through use of a laptop computer. The software for the card (FrameLink) is then used to capture and store images. A standard zoom lens (Toyo Optics 12.5-75 mm) is used and the camera is powered by a $12 \mathrm{~V}$ battery. Use of a zoom lens, rather than a wide-angle lens, reduces the parallax error. Additionally, the path that the laser light travels between the particle and camera lens is exactly the same as in standard PIV systems (particle $\rightarrow$ water $\rightarrow$ Plexiglas $\rightarrow$ air $\rightarrow$ lens), avoiding the need for additional corrections due to light refraction.

The camera depth of field is important in microscopic PIV investigations to determine the depth over which particles are tracked (Olsen and Adrian 2000, Malkiel et al 2003). In the case of field PIV systems the light sheet width (approximately $1 \mathrm{~mm}$ in this system) is the determining parameter. The depth of field does, however, determine whether unavoidable naturally occurring objects appear in the background of collected images. These background objects would show up on the PIV images as groups of static light or dark pixels reducing the calculated velocity from the cross-correlation analysis. Two steps were taken to avoid this potential error. First, a camera lens system was chosen such that the depth of field was less than $3 \mathrm{~cm}$ (aperture $=\mathrm{f} 1.8$, lens focal length $=$ $75 \mathrm{~mm}$, distance to light sheet $=1 \mathrm{~m}$ and circle of confusion $=$ $0.05 \mathrm{~mm}$; Ray 2002). Additionally, during laboratory testing the system was run with all lights turned off and field collection was conducted at night to avoid illuminating background objects.

\subsection{Laser system}

The $90 \mathrm{~mW}$ hand-held laser produces a $1.6 \mathrm{~mm}$ diameter circular beam which operates at $532 \mathrm{~nm}$ with a divergence angle of $0.9 \mathrm{mrad}$. It runs continuously once turned on via a switch on the waterproof housing. To create the sheet of light necessary to obtain PIV data, the beam is directed through a $1000 \mathrm{~mm}$ focal lens (which focuses the beam to less than $1 \mathrm{~mm}$ within the region of interest, $1000 \mathrm{~mm}$ from the lens) and then through two $12.5 \mathrm{~mm}$ cylindrical lenses to create a sheet of light. At $1000 \mathrm{~mm}$ the laser sheet has a practical width of $8 \mathrm{~cm}$. The chopper wheel is located in front of the optical lenses so that the laser beam is first chopped before being transformed into a laser sheet.

\subsection{Timing system}

The TTL signal from the camera is plugged into a microprocessor which drives a variable speed motor. The motor is attached to a chopper wheel which rotates to modulate the duration and timing of laser emission. The microprocessor allows the user to adjust the speed of the wheel, via a rheostat, to accomplish this synchronization. The microprocessor and chopper wheel are powered using a $9 \mathrm{~V}$ battery within the laser housing.

\subsection{Housing system}

The system is enclosed within two connected waterproof cases which were modified for the system. One case houses the laser, optics, beam chopper and microprocessor; the other case houses the camera. The systems are interdependent in two ways. First, the camera TTL signal is fed, via coaxial cable, between the cases through a $2 \mathrm{~m}$ hose, from the camera to the microprocessor. Second, the camera case is connected to the shore/boat via a $10 \mathrm{~m}$ hose which contains the power cord for the camera and the camera-link cable which transmits the raw images to a laptop.

The modifications made to the cases are as follows. Two holes were cut, one on each case, to allow for the hose between the cases. On the camera case there was a hole cut for the hose to the surface. There was also a hole cut in the side for the camera to capture images. The hole was covered with a square of 1/4 inch Plexiglas and siliconed in place. On the laser case, a hole was cut on the top so as to allow for the installation of a wing nut-rod system which allows one to turn the laser on/off without opening the case. Also, a hole was cut in the side of the laser case so as to insert the two cylindrical lenses and allow the laser beam to pass through the case. This was done by placing the lenses back to back inside a small o-ring and sealing the lenses into the hole.

\subsection{Post-processing}

Standard post-processing techniques may be used on the digital images (Huang et al 1997, Lang and Manglano 2004, Adrian 2005). The contrast of all images was increased using Adobe Photoshop ${ }^{\circledR}$ contrast tool in order to facilitate visual inspection of the appropriate region of interest, changing the number of outlier correlations by less than $0.3 \%$. The contrast was increased uniformly for all images so as to maintain consistency in pixel illumination between images. For the comparison in section 2 and the demonstration in section 3 , the contrast was increased by 75 contrast units for all images. Image pairs were selected which had only a single exposure per frame in two sequential frames. These image pairs were uploaded into PixelFlow, a PIV collection and post-processing software developed by General Pixels (Gharib and Dabiri 2000, General Pixels 2000). A cross-correlation algorithm is performed on $32 \times 32$ pixel windows within each image pair to determine the average particle displacement. The $32 \times 32$ pixel windows are calculated every 16 pixels across the entire region of interest to obtain a vector map of the flow. Outliers are removed if the resulting vector is \pm 3 pixels $\mathrm{s}^{-1}$ different from its neighbouring vectors (General Pixels 2000, Bartol et al 2005, Samothrakis and Cotel 2006a) and a spatial 
smoothing algorithm is employed to remove discontinuities. PixelFlow is then used to calculate the vorticity field (General Pixels 2000, Bartol et al 2003), and the vector and contour maps are plotted using TecPlot ${ }^{\circledR}$.

\section{Validation}

Validation of a new technology requires comparison of results with accepted measurement devices in canonical flows. Here we begin that process by investigating the flow behind a circular cylinder with a standard laboratory PIV and the newly developed underwater PIV system.

\subsection{Laboratory PIV system}

A PIV system developed by General Pixels (2000) was used for comparison purposes. The system consists of a $120 \mathrm{~mJ}$ NdYAG dual-head $532 \mathrm{~nm}$ pulsed laser (NewWave Gemini), timing box, 1 megapixel 10-bit, 30 fps UniqVision black-andwhite CCD camera. The camera is driven by a dedicated PC which stores the digital images. The system is run by PixelFlow software also used to post-process the image pairs. The system has been used on a variety of flows (Huang et al 1997, Gharib and Dabiri 2000, Samothrakis and Cotel 2006b) and compares well with other flow measurement tools (Westerweel et al 1997, Huang et al 1997, Liu et al 1991).

\subsection{Flow setup}

Tests were conducted in a model 29899 low turbulence closed circuit water tunnel designed by Engineering Laboratory Design, Inc. The test section of the flume is $2.44 \mathrm{~m}$ long with a square cross-section of $61 \mathrm{~cm} \times 61 \mathrm{~cm}$. Water depth was $60 \mathrm{~cm}$ and a single $1.6 \mathrm{~cm}$ diameter poly-vinyl chloride cylinder was placed horizontally in the flume at a depth of $30 \mathrm{~cm}$. Tests were conducted at cross-sectionally averaged velocities of 5.5, 9.1 and $12.9 \mathrm{~cm} \mathrm{~s}^{-1}$ which correspond to discharge-based Reynolds numbers of $3.3 \times 10^{4}, 1.4 \times 10^{5}$ and $7.9 \times 10^{5}$, respectively. The flume was seeded with $1.2 \mathrm{ppm}$ titanium dioxide particles which had a mean diameter of $1.0 \mu \mathrm{m}$ (Lin et al 2003).

\subsection{PIV setup}

The physical configurations of the underwater and laboratory PIV setups were similar. The laser sheet for each system was shone through the Plexiglas floor to illuminate the flow directly downstream of the cylinder while the camera for each system was setup perpendicular to the laser sheet to capture images of the illuminated flow. The optics for the laboratory PIV were setup such that a region approximately $30 \mathrm{~cm} \times 30 \mathrm{~cm}$ was illuminated downstream from the flow while the camera recorded a region approximately $20 \mathrm{~cm} \times$ $20 \mathrm{~cm}$ resulting in an image magnification of 33 pixels $\mathrm{cm}^{-1}$ (figure 2). The underwater PIV system necessarily illuminated a smaller region of the flow $(5 \mathrm{~cm} \times 7 \mathrm{~cm})$ due to the lower intensity of the battery-powered laser (figure 2). The camera recorded a region of $10 \mathrm{~cm} \times 10 \mathrm{~cm}$ resulting in an image magnification of 58 pixels $\mathrm{cm}^{-1}$. The pulse duration for the laboratory PIV for all tests was set to $100 \mu$ s while the pulse separations were set to 9, 8 and $4 \mathrm{~ms}$ for the Reynolds numbers of $3.3 \times 10^{4}, 1.4 \times 10^{5}$ and $7.9 \times 10^{5}$, respectively. The pulse durations for the underwater PIV were 20,6 and $6 \mathrm{~ms}$ for the Reynolds numbers of $3.3 \times 10^{4}, 1.4 \times 10^{5}$ and $7.9 \times 10^{5}$, respectively. The pulse separations for the underwater PIV were 20, 13 and $6 \mathrm{~ms}$ for the Reynolds numbers of $3.3 \times 10^{4}$, $1.4 \times 10^{5}$ and $7.9 \times 10^{5}$, respectively. The appropriate pulse separations for the underwater PIV were calculated such that the average particle displacement between image pairs would be less than one-fourth of the integration window $\left(l_{\mathrm{p}} \leqslant 0.25 l_{\mathrm{w}}\right)$ (Westerweel et al 1997, General Pixels 2000, Bernal 2003). The equation used and modified for the above units is

$$
\Delta t=\frac{250 \times \mathrm{LW}}{M \times U_{\mathrm{f}}}
$$

where $\Delta t$ is the pulse separation in milliseconds, LW is the integration window in pixels, $M$ is the magnification in pixels per centimetre and $U_{\mathrm{f}}$ is the flow speed in centimetres per second. Pulse separations for the lab PIV system were originally determined using equation (1) and subsequently shortened in order to minimize the number of outlier pixels. The pulse duration for the underwater PIV images was determined by balancing the need for increased illumination time for the low laser power system and the need to avoid particle streaking. It was determined by visual inspection of raw images that a minimum of $6 \mathrm{~ms}$ exposure was necessary to provide sufficient particle illumination. For the maximum flow inspected in this evaluation $\left(15 \mathrm{~cm} \mathrm{~s}^{-1}\right)$, the particle displacement within each pulse is approximately $0.9 \mathrm{~mm}$ (5.2 pixels). Analysis of raw images collected at $15 \mathrm{~cm} \mathrm{~s}^{-1}$ indicates an apparent particle size of approximately 5 pixels along the flow path axis by 2 pixels in the transverse axis for an average particle diameter of 3.5 pixels. Huang et al (1997) found that apparent particle size did not affect mean bias or root mean square errors for the particles that they tested (1.4 and 3.6 pixels in diameter). For the $4 \mathrm{~cm} \mathrm{~s}^{-1}$ flow, pulse durations could be increased to $20 \mathrm{~ms}$ due to the slower particle speed allowing for images to be collected using the camera shutter set to $20 \mathrm{~ms}$. Due to the greater laser power in the lab PIV system, particles could be illuminated with $100 \mu$ s pulse durations.

\subsection{Validation results}

Image pairs were collected and analysed using the methods described above. For each of the three flows, 50 image pairs (100 images) were collected using the lab PIV. One hundred images were collected at $30 \mathrm{fps}$ for each of the three flows using the underwater PIV; however, due to the nature of the chopper wheel only a portion of the 100 images corresponded to image pairs. For the Reynolds number flows of $3.3 \times 10^{4}$, the chopper wheel was not used due to the small velocities, and 40 sequential velocity pairs were used to calculate flow variables. For the quicker flows at $R e=1.4 \times 10^{5}$ and $7.9 \times 10^{5}$ where the chopper wheel was used, 17 and 21 image pairs, respectively, were used for calculations. The mean and standard deviation velocity, vorticity and average eddy diameter are reported for three flow conditions in table 1 following Lourenco et al (1994), Koutsiaris et al (1999), Perrin et al (2006) and Saikrishnan et al (2006).

The mean velocities measured by the laboratory and underwater PIV systems were comparable for each of the three 

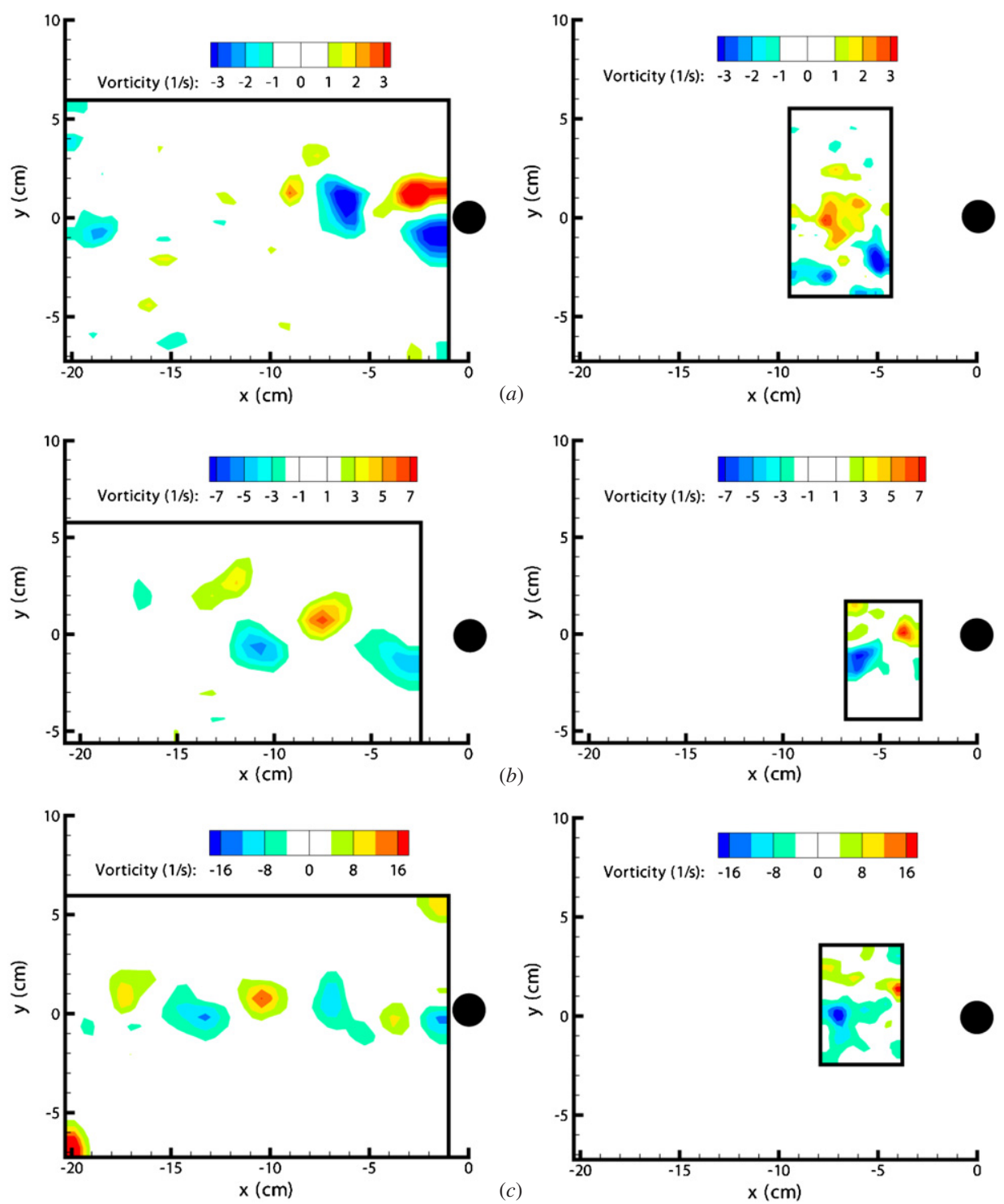

Figure 2. Selected vorticity plots from lab PIV (left) and underwater PIV (right) for the discharge Reynolds numbers of $(a) 3.3 \times 10^{4}$, (b) $1.4 \times 10^{5}$ and $(c) 7.9 \times 10^{5}$. The lab PIV illuminates a larger area than the underwater PIV. The black circle represents the obstruction cylinder; the $x$-axis is the downstream distance from the cylinder while the $y$-axis is the vertical distance from the cylinder centreline, flow is from right to left across the sheet. Vorticity scales are adjusted between flows but are the same between lab and underwater PIV images.

flows (table 1). The average velocity was calculated over the entire interrogation window of the underwater PIV and compared to the average velocity from the lab PIV calculated over the same area (figure 2). Mean velocities recorded by the underwater PIV were within $2 \%$ of the laboratory PIV values for the Reynolds numbers of $7.9 \times 10^{5}$ and $1.4 \times 10^{5}$. The $3.3 \times 10^{4}$ Reynolds number velocities measured by the underwater PIV were $0.5 \mathrm{~cm} \mathrm{~s}^{-1}(9.4 \%)$ greater than that of the laboratory PIV. The slight overestimation of velocity for the slowest flow is most likely due to the long pulse separation between images (Raffel et al 1998).

The average vorticity magnitude was also compared between systems with a maximum difference of $3.5 \%$ occurring at a Reynolds number of $7.9 \times 10^{5}$. The average vorticity magnitude was calculated over the entire region of interest of the underwater PIV and compared to the average vorticity magnitude from the lab PIV calculated over the same area. A sensitivity analysis was conducted on the effect of interrogation window size on vorticity magnitude. Doubling the underwater PIV interrogation window size from $32 \times 32$ pixels to $64 \times 64$ pixels resulted in $+1.1 \%,-2.1 \%$ and $+0.6 \%$ changes in average eddy vorticity for the $\operatorname{Re} 7.9 \times 10^{5}, 1.4 \times$ $10^{5}$ and $3.3 \times 10^{4}$ flows, respectively.

Eddies were determined by setting a threshold vorticity equal to $33 \%$ of the maximum vorticity measured by the lab PIV which corresponded to 1, 2 and $4 \mathrm{~s}^{-1}$ (figure 2). Vortex identification methods vary widely among investigations (Adrian et al 2000, Vollmers 2001, Camussi 2002) but generally produce qualitatively similar results as long as a consistent method and threshold are used across all of the data. The selection of $33 \%$ of the maximum vorticity is within the range of thresholds reported in the literature (Adrian et al 2000, Chagnaud et al 2006, Finn and Boghosian 2006) and was chosen as a threshold due to its ability to clearly identify 
Table 1. Comparisons of free stream velocity, vorticity, mean eddy diameter and maximum eddy diameter between laboratory and underwater PIV for flow downstream from a $1.6 \mathrm{~cm}$ cylinder. Reynolds numbers are based on discharge. Values in parentheses represent \pm 1 standard deviation about the mean. Note that the standard deviations are not reported for the maximum eddy diameters since they represent the single largest eddy recorded.

\begin{tabular}{lllcc}
\hline & $R e$ & Laboratory PIV & Minimum PIV & Per cent difference $(\%)$ \\
\hline Free stream velocity $\left(\mathrm{cm} \mathrm{s}^{-1}\right)$ & $7.9 \times 10^{5}$ & $15.3(2.5)$ & $15.1(3.4)$ & -1.7 \\
& $1.4 \times 10^{5}$ & $8.2(1.1)$ & $8.3(1.6)$ & +1.9 \\
& $3.3 \times 10^{4}$ & $5.2(1.2)$ & $5.7(0.9)$ & +9.4 \\
Vorticity $\left(\mathrm{s}^{-1}\right)$ & $7.9 \times 10^{5}$ & $10.77(0.81)$ & $10.40(1.16)$ & -3.5 \\
& $1.4 \times 10^{5}$ & $4.41(0.60)$ & $4.31(0.80)$ & -2.1 \\
& $3.3 \times 10^{4}$ & $1.72(0.22)$ & $1.69(0.23)$ & -1.5 \\
Mean eddy diameter $(\mathrm{cm})$ & $7.9 \times 10^{5}$ & $1.3(0.8)$ & $1.0(0.8)$ & -24.8 \\
& $1.4 \times 10^{5}$ & $1.7(0.8)$ & $1.1(0.9)$ & -36.6 \\
& $3.3 \times 10^{4}$ & $1.1(0.8)$ & $0.9(0.8)$ & -17.0 \\
Max Eddy diameter $(\mathrm{cm})$ & $7.9 \times 10^{5}$ & 3.1 & 3.3 & +5.9 \\
& $1.4 \times 10^{5}$ & 3.5 & 3.0 & -14.0 \\
& $3.3 \times 10^{4}$ & 3.2 & 3.8 & +20.1 \\
\hline
\end{tabular}

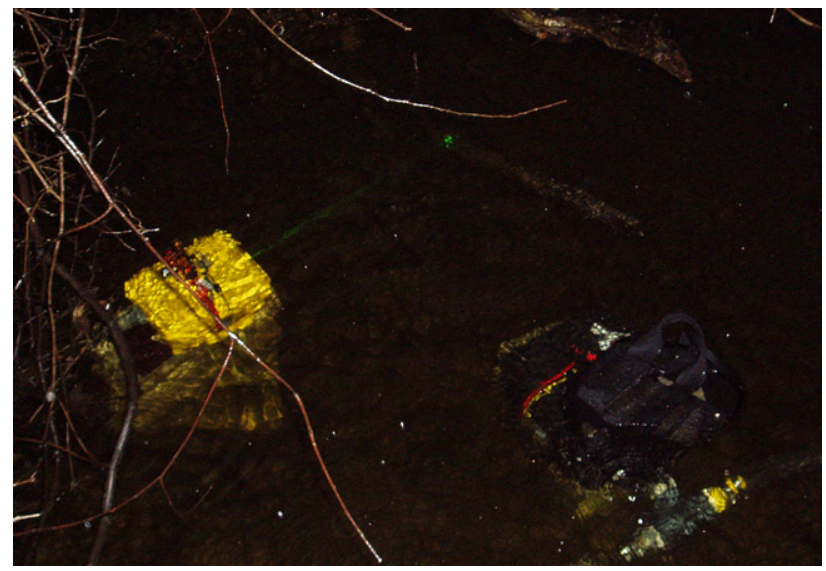

Figure 3. Image of underwater PIV being used in the Huron River, MI.

individual eddies. Eddy diameters were determined by calculating eddy area and equivalent eddy diameter assuming that eddies have a 1-to-1 aspect ratio. Average eddy diameters measured by the underwater PIV were on average $26 \%$ less than the eddy diameters measured by the laboratory PIV. This is due to the smaller interrogation region available to the underwater PIV compared to the lab PIV. The smaller area of interrogation for the underwater PIV resulted in more partial eddies (i.e. the underwater illuminated a smaller area; therefore many eddies were only partially captured causing a smaller recorded diameter). The average difference for the maximum eddy size is $4 \%$ across the three runs indicating consistency when only complete eddies are included.

\section{Field demonstration}

The underwater PIV was field tested in the Huron River, Ann Arbor, MI on 10 January 2007 (figure 3). Average discharge during the data collection was $40.0 \mathrm{~m}^{3} \mathrm{~s}^{-1}$ and varied less than $2 \%$ during the data collection period. Flow data just downstream from a $1.2 \mathrm{~cm}$ diameter limb, just upstream $(10 \mathrm{~m})$ from USGS gauging station 04174500 were collected. Natural
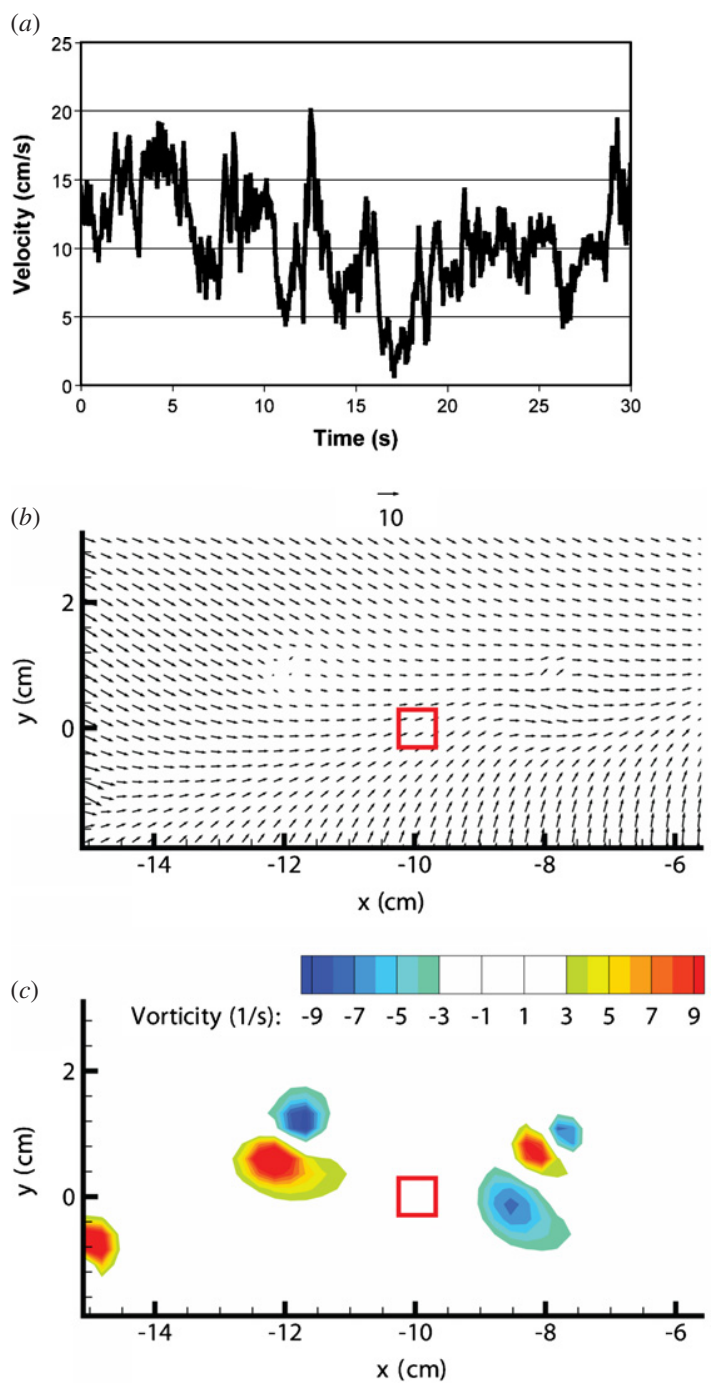

Figure 4. Field data. (a) ADV time series-streamwise velocity plotted; $(b)$ instantaneous vector field from underwater PIV-reference vector is $10 \mathrm{~cm} \mathrm{~s}^{-1}$; (c) instantaneous vorticity field from underwater PIV. The limb is located at $x=0 \mathrm{~cm}$ and the ADV control volume is indicated with a $1 \mathrm{~cm}^{2}$ red box at $x=-10 \mathrm{~cm}$, $y=0 \mathrm{~cm}$. 
particles suspended in the Huron River were sufficient for flow illumination during field collection. Errors due to low particle density have been shown to be significantly reduced when illuminated particle concentrations are greater than 10 per interrogation window (Willert and Gharib 1991, Keane and Adrian 1992, Bertuccioli et al 1999). Water collected during field measurements had a turbidity of 37 NTU and had a concentration of $34.8 \mathrm{mg} \mathrm{L}^{-1}$ resulting in an average of 16 illuminated particles per interrogation window. The PIV system was set up such that the laser system was $1 \mathrm{~m}$ downstream and the camera system was $1.2 \mathrm{~m}$ transverse to the region of interest (figure 3). This configuration ensured that any disturbances to the flow were convected downstream rather than into the region of interest. The two systems were set perpendicular to each other using right-angle squares though the use of a rigid arm connector would simplify future field setups. Fifteen image pairs were collected with the underwater PIV over a single $3.3 \mathrm{~s}$ (100 images) data collection event; pulse separation and duration were set to $7 \mathrm{~ms}$ and image magnification was 33 pixels $\mathrm{cm}^{-1}$.

Point velocity data were collected with an ascoustic Doppler velocimeter (ADV) in addition to the underwater PIV (figure 4). The ADV was set up $10 \mathrm{~cm}$ downstream from the submerged limb and data were collected at a frequency of $30 \mathrm{~Hz}$ for $2 \mathrm{~min}$. Two $2 \mathrm{~min}$ time series were collected at the same location $15 \mathrm{~min}$ apart.

PIV parameters were calculated using the techniques described above. Average velocities were calculated for the $0.36 \mathrm{~cm}^{2}$ area corresponding to the location of the ADV sample volume. Average velocities measured by the underwater PIV and ADV were 11.0 and $10.8 \mathrm{~cm} \mathrm{~s}^{-1}$, respectively. The recorded temporal standard deviations of the velocity fluctuations were 5.0 and $4.8 \mathrm{~cm} \mathrm{~s}^{-1}$ for the PIV and ADV systems, respectively. The average eddy size recorded by the underwater PIV was determined to be $0.79 \mathrm{~cm}$ and the average vorticity magnitude was $4.0 \mathrm{~s}^{-1}$.

\section{Conclusions}

This newly developed underwater and highly portable PIV system has been shown to provide comparable velocity, vorticity field and eddy length scales to a standard laboratory PIV system under low to moderate velocity conditions. Tests so far provide high confidence in the underwater PIV results for instantaneous velocities up to $15 \mathrm{~cm} \mathrm{~s}^{-1}$ which is a practical range for many lake and low gradient river systems, and boundary layer regions. Velocity measurements from the underwater PIV were within $10 \%$ of those recorded by a standard laboratory PIV while vorticity measurements were within $3.5 \%$. Average eddy sizes recorded by the underwater PIV system were $26 \%$ less than those recorded by the laboratory PIV due to eddy cropping and magnification effects. The system cost $\$ 7500$ to develop (including laptop but excluding post-processing software which can be developed by a user or purchased in addition to the system) and field data in the Huron River were collected using two people over $3 \mathrm{~h}$, making the system comparable to existing ADV systems in price and portability. The spatial velocity maps from the underwater PIV can be used to calculate vorticity and eddy diameters with greater spatial resolution than previously published ADV investigations. The current system can be modified for a larger illumination region by incorporating a $300 \mathrm{~mW}$ laser rather than the $90 \mathrm{~mW}$ laser used in this study. A stronger laser coupled with shorter chopping times would also increase the range of velocities attainable. The underwater PIV was originally designed for investigations of ecologically relevant turbulence in natural environments. Other obvious uses include investigation of flow over naturally occurring bed forms and groundwater flow into surface water bodies. Another anticipated use is to validate theoretical and numerical models for unsteady flow in natural aquatic systems.

\section{Acknowledgments}

This work was partially supported by the National Science Foundation under grant CTS-0447427, the Michigan Sea grant programme under grant R/GLF-53 and research equipment funds from the Department of Civil and Environmental Engineering at the University of Michigan. We are grateful to Paul Webb for his feedback and Dana Dabiri for his advice during the instrument development stage. We would also like to thank Pratik Pradhan and Jun Lee for their laboratory assistance.

\section{References}

Adrian R J 2005 Twenty years of particle image velocimetry Exp. Fluids 39 159-69

Adrian R J, Christensen K T and Liu Z C 2000 Analysis and interpretation of instantaneous turbulent velocity fields Exp. Fluids 29 275-90

Bartol I K, Gharib M, Webb P W, Weihs D and Gordon M S 2005 Body-induced vortical flows: a common mechanism for self-corrective trimming control in boxfishes J. Exp. Biol. 208 327-44

Bartol I K, Gharib M, Weihs D, Webb P W, Hove J R and Gordon M S 2003 Hydrodynamic stability of swimming in ostraciid fishes: role of the carapace in the smooth trunkfish Lactophrys triqueter (teleostei: ostraciidae) J. Exp. Biol. $206725-44$

Bernal L P 2003 Experimental Methods in Fluid Mechanics L3.5 (Course Notes) University of Michigan Course \# Aerosp 521

Bertuccioli L, Roth G I, Katz J and Osborn T R 1999 A submersible particle image velocimetry system for turbulence measurements in the bottom boundary layer J. Atmos. Ocean. Technol. 16 1635-46

Camussi R 2002 Coherent structure identification from wavelet analysis of particle image velocimetry data Exp. Fluids 32 76-86

Chagnaud B P, Bleckmann H and Engelmann J 2006 Neural responses of goldfish lateral line afferents to vortex motions J. Exp. Biol. 209 327-42

Chetelat O, Yoon S Y and Kim K C 2001 Design and construction of a miniature PIV (MPIV) system Korean Soc. Mech. Eng. J. 15 1775-83

Dabiri D 2003 On the interaction of a vertical shear layer with a free surface J. Fluid Mech. 480 217-32

Finn L I and Boghosian B M 2006 A global variational approach to vortex core identification Physica A 362 11-6

General Pixels 2000 PixelFlow 2.1: Installation and User's Guide (Pasadena, CA)

Gharib M and Dabiri D 2000 An overview of digital particle image velocimetry Flow Visualization: Techniques and Examples ed A Smits and T T Lim (London: Imperial College Press)

Huang H, Dabiri D and Gharib M 1997 On errors of digital particle image velocimetry Meas. Sci. Technol. 8 1427-40 
Keane R D and Adrian R J 1992 Theory of cross-correlation analysis of PIV images Appl. Sci. Res. 49 191-215

Koutsiaris A G, Mathioulakis D S and Tsangaris S 1999 Microscope PIV for velocity-field measurement of particle suspensions flowing inside glass capillaries Meas. Sci. Technol. 10 1037-46

Landry F, Miller T J and Leggett W C 1995 The effects of small-scale turbulence on the ingestion rate of fathead minnow larvae Can. J. Fish Aquatic Sci. 52 1714-9

Lang A W and Manglano C E 2004 An experimental study of a turbulent shear layer at a clean and contaminated free-surface Exp. Fluids 36 384-92

Lin C, Lai W J and Chang K A 2003 Siumltaneous particle image velocimetry and laser doppler velocimetry measurements of periodical oscillatory horseshoe vortex system near square cylinder-base plate juncture J. Eng. Mech. 129 1173-88

Liu Z C, Landreth C C, Adrian R J and Hanratty T J 1991 High resolution measurement of turbulent structure in a channel with particle image velocimetry Exp. Fluids 10 301-12

Lourenco L M, Sivram P G and LaSalle R T 1994 On-line particle-image velocimeter: an integrated approach Appl. Optics 33 2465-70

Malkiel E, Sheng J, Katz J and Strickler J R 2003 The three-dimensional flow field generated by a feeding calanoid copepod measured using digital holography J. Exp. Biol. $2063657-66$

Nikora V I, Aberlee J, Biggs B J F, Jowett I G and Sykes J R E 2003 Effects of fish size, time to fatigue, and turbulence on swimming performance: a case study of Galaxias maculatus J. Fish Biol. 63 1365-82

Nimmo-Smith W A M, Atsavapranee P, Katz J and Osborn T R 2002 PIV Measurements in the bottom boundary layer of the coastal ocean Exp. Fluids 33 962-71
Olsen M G and Adrian R J 2000 Out-of-focus effects on particle image visibility and correlation in microscopic particle image velocimetry Exp. Fluids Suppl. S166-74

Perrin R, Braza M, Cid E, Cazin S, Moradei F, Barthet A, Sevrain A and Hoarau Y 2006 Near-wake turbulence properties in a high Reynolds number incompressible flow around a circular cylinder measured by two and three component PIV Flow Turb. Combust. 77 185-204

Raffel M, Willert C and Kompenhans J 1998 Particle Image Velocimetry: A Practical Guide (Berlin: Springer)

Ray S F 2002 Applied Photographic Optics (Oxford: Focal Press)

Roy A G, Buffin-Belanger T, Lamarre H and Kirkbride A 2004 Size, shape, and dynamics of large scale turbulent flow structures in a gravel bed river J. Fluid Mech. 500 1-27

Saikrishnan N, Marusic I and Longmire E K 2006 Assessment of dual plane PIV measurements in wall turbulence using DNS data Exp. Fluids $\mathbf{4 1}$ 265-78

Samothrakis P and Cotel A J 2006a Finite volume gravity currents impinging on a stratified interface Exp. Fluids 41 991-1003

Samothrakis P and Cotel A J 2006b Propagation of a gravity current in a two-layer stratified environment J. Geophys. Res. $111 \mathrm{C} 01012$

Standen E M, Hinch S G and Rand P S 2004 Influence of river speed on path selection by migrating adult sockeye salmon Can.J. Fish Aquatic Sci. 61 905-12

Vollmers H 2001 Detection of vortices and quantitative evaluation of their main parameters from experimental velocity data Meas. Sci. Technol. 12 1199-207

Westerweel J, Dabiri D and Gharib M 1997 The effect of a discrete window offset on the accuracy of cross-correlation analysis of digital PIV recordings Exp. Fluids $2320-8$

Willert C E and Gharib M 1991 Digital particle image velocimetry Exp. Fluids 10 181-93 